\title{
THE IMPACT OF CONSUMER INNOVATIVENESS, PRESTIGE PRICE SENSITIVITY AND NEED FOR EMOTION ON IMPULSE BUYING AND SATISFACTION
}

\author{
Dr. Lukman Aroean, Bournemouth University, UK \\ Dr. Nina Michaelidou, Loughborough University, UK
}

\section{INTRODUCTION}

Impulse buying (IB) refers to unplanned purchases without much reflection (Bellenger et al. 1978; Youn and Faber 2000; Beatty and Ferrell, 1998) and it is defined by Rock (1987: 191) as a state of "sudden, often powerful and persistent urge to buying something immediately". Commonly conceptualized and measured in the literature as a tendency to buy things on impulse (Rook and Fisher 1995; Peck and Childers 2006), IB is "inconsistent with rational choice models" (Silvera, et al. 2008: 23) and has been linked to affect and emotion, specifically excitement, pleasure and an urge to buy (Rook 1987). Silvera et al. (2008) argue that there is relatively little research on IB from a psychological perspective and hence a lack of understanding of the psychological constructs underpinning this behavioral tendency. Earlier research failed to provide personalitygrounded explanations of IB (e.g. d' Antoni and Shenson, 1973; Cobb and Hoyer 1986; Youn and Faber 2000) suggesting that this stream of research may have focused on relationships between IB and 'irrelevant' personality traits (Youn and Faber 2000). Later research has, however, linked IB to a number of personality traits, specifically, lack of control, stress reaction, absorption (Youn and Faber 2000) as well as the Big 5 (Verplanken and Herabadi 2001). Additionally, Silvera et al.'s (2008) research attempts to validate Verplanken et al.'s (2005) contribution on the psychological states underlying IB, highlighting the relationship between IB and subjective wellbeing, affect, self-esteem and social influence. Lately, research has examined IB in relation to variety seeking tendencies, optimal stimulation level and price consciousness/sensitivity (Sharma et al. 2010; Liang 2012), and to other factors, such as product involvement and product knowledge, (Sharma et al. 2010), store promotions and environmental cues (Kacen et al. 2012; Peck and Childers 2006). IB has also been linked to satisfaction in the sense that consumers do not necessarily view IB as normatively wrong; but use IB as a means of satisfying certain needs, in addition to the need for the product (e.g. Hausman 2000) such as hedonic desires, novelty, variety, social interaction and self-esteem needs (Piron 1991; Rook 1987; Hausman 2000). This study seeks to extend the current understanding of the underpinnings of IB and satisfaction by focusing on specific personality traits that seem more relevant for marketers, including consumer innovativeness, prestige price sensitivity and need for emotion.

\section{BACKGROUND AND THEORETICAL MODEL}

Consumer innovativeness (CI) is a personality trait involving individual differences in response to new products (Goldsmith and Hofacker 1991; Midgley and Dowling 1978), and defined as a tendency or disposition to buy new products faster than other consumers (Midgley and Dowling 1978). CI is linked to cognition and sensation seeking (Venkatraman and Price 1990) suggesting that cognitive innovators prefer a greater amount of information and are price-sensitive, while sensory (or emotional) innovators are more interested in pleasure and novelty (Park et al. 2010). Roehrich (2004) suggests that a number of underlying needs explain consumer 'innate' innovativeness such as the need for stimulation, variety seeking tendency (Raju 1980), novelty seeking and creativity (Hirschman 1980) as well as the need for uniqueness (Gatignon and Robertson 1985), indicating that $\mathrm{CI}$ and IB have a common underlying theoretical platform, and suggesting that the two concept maybe related.

Further, previous work suggests that emotional states (e.g. need for emotion) with a varied degree between individuals, may have behavioral consequences such as IB (Cohen and Andrade 2004; Weinberg and Gottwald 1982) and consumers seem to be aware of this motivational effect (Rook and Gardner 1993). Need for emotion (NFE) is an enduring trait (Lee et al 2009; Roehm Jr. and Roehm 2005), that refers to the tendency or disposition to see affective stimuli and enjoy emotionally-laden situations (Raman et al 1995), irrespective of the strength of the experiential emotion per se. NFE is relevant to the 'sensory' element of CI (Venkatraman and Price, 1990; Park et al. 2010) suggesting that innovative individuals are disposed to experience emotions in their interactions with marketing stimuli including new stimuli (Raman et al 1995). Similarly, NFE could be related to IB since, in line with literature, IB is linked to affect and emotions such as pleasure and excitement (Rook 1987). It is therefore suggested that NFE may underpin IB, and that consumers act on impulse in their purchases in order to also satisfy their NFE.

Another construct relevant to IB is prestige price sensitivity (PPS), which refers to consumers' belief that the purchase of the most expensive brand is a positive experience (Lichtenstein et al. 1993) that impresses others (Lichtenstein et al 1993; Netemeyer et al 1995). Lichtenstein et al. (1993) view PPS as "a favorable perception 
of the price cue based on feelings of prominence and status that higher prices signal to other people about the purchaser" (p. 236). Previous, yet limited, research has linked IB to sensitivity to price (e.g. Liang 2012), without discriminating between price cues (e.g. prestige, value etc.), suggesting that price sensitivity impacts the relationship between product involvement and IB. Price sensitivity is also linked to CI, with Goldsmith and Newell (1997) suggesting that innovative consumers are price insensitive, while Park et al. (2010) argue that cognitive innovative consumers are price conscious as opposed to sensory innovative consumers. This study focuses on the 'prestige' element of price (see Lichtenstein et al. 1993), and argues that PPS impacts IB. On this basis, consumers act on impulse in their purchases to satisfy their need to impress others.

Based on the extant literature, a model has been developed (figure 1) showing the relationships among NFE, PPS and CI with IB and satisfaction.

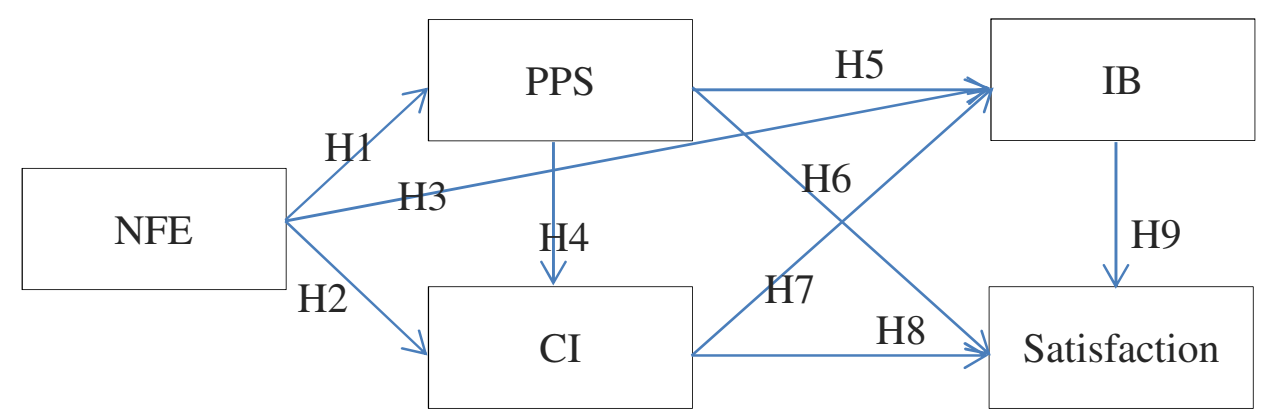

Figure 1 A Relationship model of personality traits, impulse buying and satisfaction

\section{METHOD}

A questionnaire was used to collect data from a sample of 304 respondents in a metropolitan city in the UK. Respondents were asked to complete a set of questions measuring the model's constructs. In doing so, respondents were instructed to choose a product of interest for which IB likely to occur. The products selected by the participants ranged from electronic items (e.g. games machines/consoles, mobile phones, mp3 and music players, computers, and digital cameras) to non-entertainment household products, sporting goods, clothing, accessories, food and newspapers. Existing measures were used to capture product category specific CI (Goldsmith and Hofacker 1991) and product category specific IB (Donthu and Gilliland 1996). PPS was measured by a nine-item scale from Lichtenstein et al. (1993), while a twelve-item scale from Raman et al. (1995) was used to measure NFE. Finally, satisfaction was measured using a four-item scale developed by Spreng, et al. (1996).

\section{RESULTS}

We used AMOS 19 to run CFA to assess the reliability and validity of the scales. The overall fit of the CFA is good: $\chi^{2}{ }_{(395)}=807.1 ;$ rmsea $=.059 ; \mathrm{TLI}=.92 ; \mathrm{CFI}=.92(\mathrm{Hu}$ and Bentler 1999). All constructs have both alpha and construct reliability (CR) values above .7, and most are with AVE .5 or above, with the lowest AVE very close to .5. Also, all items' loading are significant $(\mathrm{p}<.001)$ and substantive (standardized loading .5 or above, Hair et al 2010: 709) demonstrating the convergent validity of the measures. All AVEs are greater than the squared correlation coefficients between corresponding constructs, providing good evidence of discriminant validity of the measurements (Hair et al 2010).

The SEM analysis produced good fit results: $\chi^{2}{ }_{(400)}=872.0 ; \mathrm{rmsea}=.062 ; \mathrm{TLI}=.91 ; \mathrm{CFI}=.91(\mathrm{Hu}$ and Bentler 1999). The results give support for 4 of the 9 hypotheses (table 1). NFE only influences CI (H3: $\beta=.14$, $p<.05$ ), and does no predict PPS (H1) and IB (H3). PPS positively influences CI (H4: $\beta=.32, \mathrm{p}<.001)$, and positively influences IB (H5: $\beta=.25, p<.001)$, but does not predict Satisfaction (H9). Only CI positively influences satisfaction (H8: $\beta=.30, \mathrm{p}<.001)$, while PPS (H6) and IB (H9) do not.

Table 1: SEM results

\begin{tabular}{|l|c|c|c|}
\hline \multicolumn{1}{|c|}{ Hypotheses } & $\begin{array}{c}\text { Standardized } \\
\text { path coefficient } \\
(\beta)^{\mathrm{a}}\end{array}$ & $\begin{array}{c}\text { Hypothesis } \\
\text { supported? }\end{array}$ & $\begin{array}{c}\text { Standardized } \\
\text { path coefficient } \\
(\beta)^{\mathrm{b}}\end{array}$ \\
\hline H1: NFE $\rightarrow$ PPS & -.10 & No & $-\cdot-\cdot-\cdot-\cdot-\cdot-\cdot---$ \\
\hline H2: NFE $\rightarrow$ CI & $.14^{*}$ & Yes & $.13^{*}$ \\
\hline H3: NFE $\rightarrow$ IB & -.12 & No & $-\cdot---\cdot---\cdot----$ \\
\hline
\end{tabular}




\begin{tabular}{|c|c|c|c|}
\hline $\mathrm{H} 4: \mathrm{PPS} \rightarrow \mathrm{CI}$ & $.32 * * *$ & Yes & $.31 * * *$ \\
\hline H5: PPS $\rightarrow$ IB & $.25 * * *$ & Yes & $.26 * * *$ \\
\hline H6: PPS $\rightarrow$ Satisfaction & -.07 & No & 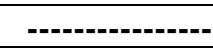 \\
\hline $\mathrm{H} 7: \mathrm{CI} \rightarrow \mathrm{IB}$ & .08 & No & |--------------- \\
\hline H8: CI $\rightarrow$ Satisfaction & $.30 * * *$ & Yes & $.27 * * *$ \\
\hline H9: IB $\rightarrow$ Satisfaction & .05 & No & 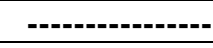 \\
\hline
\end{tabular}

$* * * \mathrm{p}<.001 ; * * \mathrm{p}<.01 ; * \mathrm{p}<.05^{\mathrm{a}}$ Full model ${ }^{\mathrm{b}}$ Re-specified model

A re-specified model (removing non-significant paths) was run with the insignificant path coefficients removed, shows good fit: $\chi_{(405)}^{2}=879.0 ;$ rmsea $=.062 ; \mathrm{TLI}=.91 ; \mathrm{CFI}=.91$. The significant path coefficients are steady for both the original and the re-specified model. The results show that $\mathrm{CI}$ is the only predictor of satisfaction and that the construct is well explained by CI with a medium effect size. The results also suggest that CI mediates NFE and PPS influence on satisfaction. Mathematically, the effect of NFE and PPS over satisfaction is .042 and .096 respectively (Satisfaction $=.30$ Innovativeness $=.30 *(.14 \mathrm{NFE}+.32 \mathrm{PPS})=.042 \mathrm{NFE}+.096 \mathrm{PPS})$. While other constructs in our model (NFE, PPS and CI) explain satisfaction either directly or indirectly, IB does not, implying that IB does not provide the satisfaction for the individual. Further, PPS in is the only construct that explains IB well with a medium effect size. This finding expands our knowledge of the role of prestige, particularly in activating impulse buying.

\section{DISCUSSION AND CONCLUSION}

The study reveals the impact of PPS on IB, while NFE and CI do not influence IB. This finding indicates that within the context of individual consumer's product of interest, IB is related to prestige, but not to general affective stimuli, and not to the tendency to buy new products faster than other consumers. Therefore, when it comes to impulsively buying a product of interest, an individual tends to be sensitive to the 'prestige' cue of product prices; and is not driven by the need for emotive stimuli nor innovativeness. This finding subtly implies that while IB is inconsistent with rational choices (Silvera et al. 2008) and therefore linked to emotion, the individual engaging in impulse buying neither does so out of emotion, nor his/her decision is based on emotion or affect. Likewise, while IB reflects a 'rushed' act of buying, the individual is not necessarily innovative within a specific product domain.

Further, the study shows that IB does not necessarily lead to satisfaction, a finding that refutes previous research (Hausman 2000). In contrast, findings show that satisfaction is determined by CI, with CI also mediating the impact of PPS on satisfaction and NFE enhancing CI with low effect. This finding further contributes to our understanding of the direct and indirect influences of CI, PPS and NFE on satisfaction.

The above findings and implications need to be carefully considered, as the context of the study is concerned with respondents' own product of interest, which signify the likelihood of IB to occur. Future research may apply the research model for a specific product category, with contrasting specific prestigious and emotive stimuli.

\section{REFERENCES available upon request}

